\title{
L'oncogène v-erbA : un inhibiteur d'inhibiteur
}

Durant leur différenciation, les cellules doivent coordonner leur activité proliférative et l'expression d'un programme génétique nouveau. On constate que, dans la plupart des tissus, la différenciation des cellules s'accompagne de la restriction de leur activité mitotique. Par ailleurs, on a observé, dans de nombreux modèles tissulaires, que l'engagement des cellules dans un programme morphogénétique spécifique ne peut se faire que durant une fenêtre de repos mitotique, généralement en phase G1. Ces données suggèrent l'existence de gènes maîtres dont les produits coordonneraient les programmes génétiques de la division et de la différenciation, par exemple en activant et réprimant simultanément la transcription de groupes de gènes impliqués respectivement dans ces programmes.

Les gènes qui codent pour les récepteurs de l'hormone thyroïdienne T3 et pour les récepteurs de l'acide rétinoïque appartiennent vraisemblablement à cette catégorie de gènes maîtres. En effet, chez les vertébrés, l'hormone T3 et l'acide rétinoïque sont deux médiateurs très importants dans la morphogenèse de certains tissus au cours du développement. Les récepteurs de ces médiateurs sont des facteurs de transcription appartenant à la superfamille des récepteurs hormonaux nucléaires [1] $\left(m / s n^{\circ} 3\right.$, vol. 3, p. 172 et $n^{\circ} 3$, vol. 4, p. 196). Différentes isoformes des récepteurs de T3 et de l'acide rétinoïque ont été clonées et il apparaît que ces deux familles de récepteurs sont fonctionnellement et structurellement très apparentées. Certaines formes du récepteur T3 sont codées par le protooncogène $c$-erb $A$ dont la version oncogénique $v$-erbA a été identifiée dans le génome d'un rétrovirus leucémogène aviaire, le virus de l'érythroblastose aviaire (AEV) [1]. Dans ce virus, l'oncogène $v$-erbA est associé à l'onco- altérée du récepteur de membrane du facteur de croissance EGF (epidermal growth factor). L'expression combinée de ces deux oncogènes dans les cellules infectées est nécessaire à la transformation néoplasique [2].

Nous avons montré que l'oncogène $v$ erbA agissant seul altère les programmes de multiplication et de différenciation de certaines cellules. Il active la prolifération des fibroblastes embryonnaires de poulet et contribue par là au développement de sarcomes in vivo [3]. Il bloque le programme de différenciation des précurseurs érythrocytiques et maintient ces cellules dans un cycle d'auto-renouvellement [4]. Ces observations suggéraient que $v$-erb $A$ interfère avec des mécanismes centraux de contrôle des programmes de différenciation et de prolifération cellulaire.

La protéine codée par l'oncogène v-erbA est très largement réarrangée par rapport à son homologue normal codé par $c$-erbA. En particulier, v-ErbA ne peut fixer l'hormone T3 [1]. On sait maintenant que cette protéine se comporte comme un inhibiteur compétitif à la fois de c-ErbA et des récepteurs de l'acide rétinoïque pour la régulation de la transcription de gènes cibles $(\mathrm{m} / \mathrm{s}$ $n^{\circ} 8$, vol. 5, p. 601) [5-8]. Ces effets de l'oncoprotéine pourraient résulter, soit d'une compétition directe avec les récepteurs normaux pour la fixation au niveau de séquences de régulation des gènes cibles, soit de la formation d'hétérodimères inactifs avec ces récepteurs. Aucune de ces deux hypothèses n'a encore reçu de démonstration absolue. Un des mécanismes d'action de l'oncogène v-erbA est donc d'interférer directement avec le contrôle de la transcription de certains gènes par $\mathrm{T} 3$ et l'acide rétinoïque.

Afin d'expliquer les effets de verbA sur la croissance des fibroblastes, nous avons supposé que l'oncoprotéine altérait de la même façon une réponse proliférative de ces cellules à $\mathrm{T} 3$ ou à l'acide rétinoïque. En fait, nous n'avons pas observé d'effet significatif de T3 sur la croissance des fibroblastes de poulet, vraisemblablement parce que ces cellules possèdent très peu de récepteurs de l'hormone. En revanche, nous avons observé que l'acide rétinoïque bloque la prolifération de ces cellules en altérant des processus moléculaires en début de phase $\mathrm{G} 1$, et que l'expression de $v$-erbA dans ces cellules lève cette inhibition [9]. Il fut très intéressant de constater que deux autres oncogènes nucléaires, les oncogènes $v$ fos et $v$-jun, montrent des effets identiques à ceux de verbA dans ce modèle. Les protéines v-Fos et v-Jun sont des formes altérées des protéines c-Fos et c-Jun qui entrent dans la composition du complexe de transcription AP-1 [10]. Cette observation nous suggéra immédiatement que la protéine $\mathrm{v}$ ErbA pourrait interférer fonctionnellement avec le complexe AP-1. Cette hypothèse trouva un très fort soutien quand d'autres groupes et nous-mêmes montrèrent que les récepteurs de $\mathrm{T} 3$ et de l'acide rétinoïque inactivent le complexe de transcription AP-1 et, par voie de conséquence, réduisent au silence des gènes dont la transcription est dépendante de AP-1 [11-13]. Il apparaît ainsi que les récepteurs de T3 et de l'acide rétinoïque peuvent contrôler l'expression génétique par deux voies distinctes qui sont schématisées sur la figure $1 A$. Ce schéma permet d'imaginer comment deux groupes de gènes (marqués respectivement I et II sur la figure) peuvent voir leurs expressions modulées de façons opposées en réponse à une stimulation hormonale. Parmi les gènes du groupe II activés par la voie classique décrite initialement, on trouve de nombreux gènes impliqués dans le développement d'un programme de différenciation, comme par exemple des gènes homéo- 


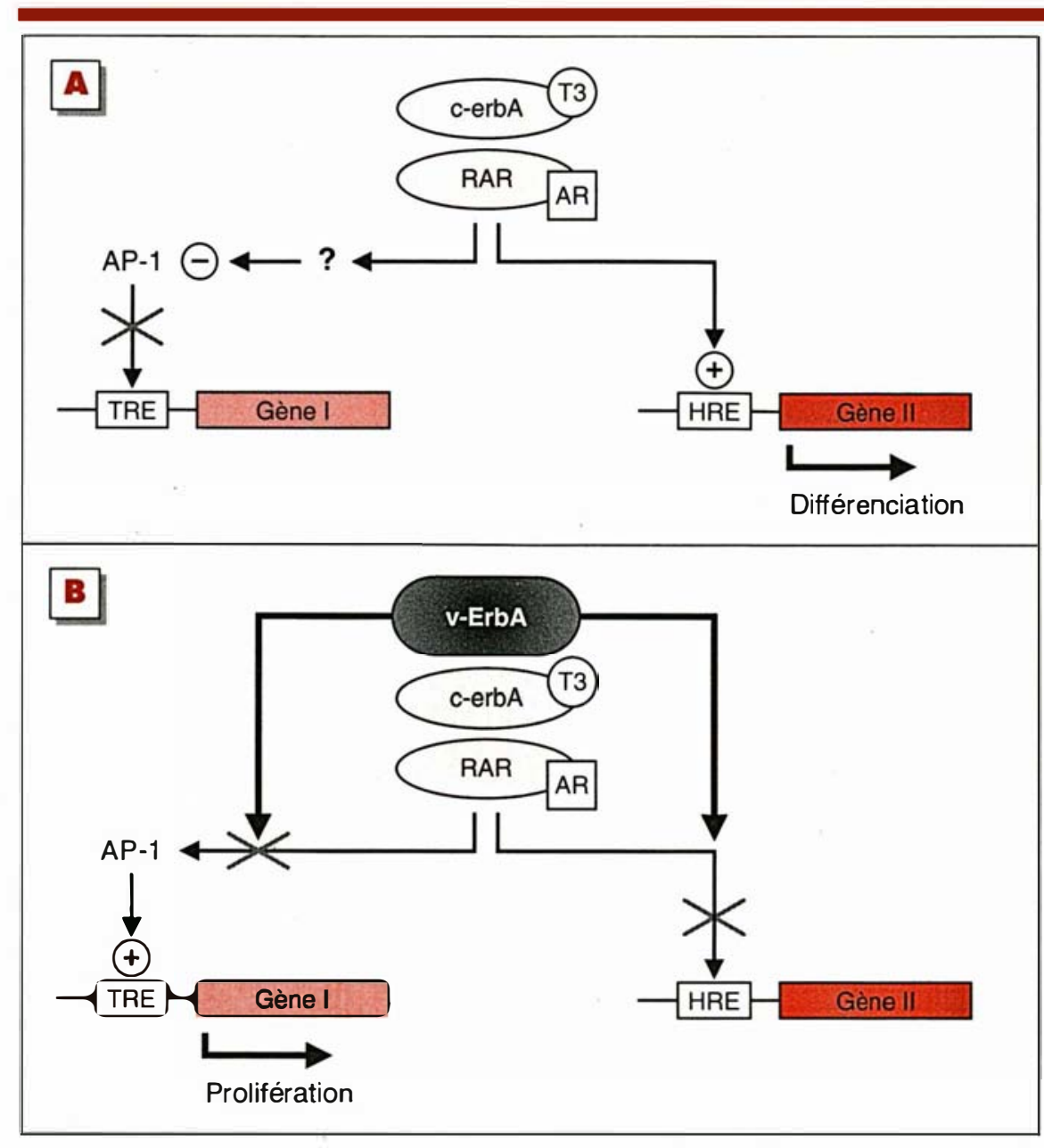

Figure 1. Schéma montrant la dualité fonctionnelle des récepteurs de T3, de l'acide rétinoïque et de la protéine v-ErbA. Sur la figure $1 \mathrm{~A}$ on a représenté le fonctionnement des récepteurs de T3 et de l'acide rétinoïque au cours du développement de la cellule normale. Les récepteurs activent la transcription de gènes du groupe II par l'intermédiaire d'une séquence de réponse HRE (hormone response element). En parallèle, ces récepteurs inactivent le facteur de transcription AP-1 qui est incapable d'induire la transcription des gènes du groupe I par l'intermédiaire de la séquence de fixation de AP-1 appelée TRE (TPA response element). Sur le schéma, on considère que les gènes impliqués dans l'expression d'un programme de différenciation appartiennent au groupe II et que les gènes de groupe I renferment des gènes impliqués dans l'activité proliférative de la cellule. Sur la figure 1B, on a représenté la situation d'une cellule qui exprime l'oncoprotéine $v$-ErbA. La protéine $v$-ErbA se comporte en antagoniste des deux voies de régulation contrôlées par les récepteurs. Cet effet entraîne l'inhibition des gènes du groupe II et le maintien en activité des gènes du groupe I. (RAR : récepteur de l'acide rétinoïque; $A R$ : acide rétinoïque.)

tiques dans le bourgeon de membre, le gène de l'anhydrase carbonique II dans les érythroblastes, le gène de la chaîne lourde de myosine dans les myoblastes. Le complexe AP-1 joue un rôle déterminant dans la multiplication cellulaire, vraisemblablement en activant l'expression de gènes dont les $m / s n^{\circ} 2$, vol. 8, fériar 92 ment enclencher un programme de différenciation et freiner la prolifération. $\mathrm{Au}$ vu de la dualité fonctionnelle de ces récepteurs, il apparaissait extrêmement intéressant d'analyser le comportement de la protéine v-ErbA dans ce modèle. Nous avons trouvé que l'oncoprotéine est incapable d'inactiver AP-1 et que, en outre, elle peut exercer un effet dominant sur les récepteurs de T3 et de l'acide rétinoïque en neutralisant l'action inhibitrice de ces récepteurs sur AP-1 [11]. La protéine v-ErbA se comporte donc comme un inhibiteur d'inhibiteur. On conclut donc que dans des cellules qui produisent $\mathrm{v}$ ErbA, l'activité AP-1 n'est pas diminuée en présence de $\mathrm{T} 3$ ou d'acide rétinoïque. Afin de tester la signification physiologique de cette observation, nous avons analysé le comportement de divers mutants de $v$-erbA et de $c$-erbA et nous avons observé une corrélation inverse très nette entre leur capacité à inactiver AP-1 et leur aptitude à promouvoir la croissance de fibroblastes en culture. Ces analyses suggèrent fortement que l'interférence fonctionnelle entre AP-1, les récepteurs normaux et $\mathrm{v}$-ErbA rend compte des effets sur la prolifération cellulaire.

Ces travaux mettent donc à jour une dualité de fonction de l'oncoprotéine vErbA qui est schématisée sur la figure 1B. L'oncoprotéine inhibe la transcription des gènes du groupe II, et, à l'inverse, maintient en activité les gènes du groupe I. On conçoit ainsi comment v-ErbA agit simultanément en inhibant la différenciation et en favorisant la prolifération des cellules, deux effets qui sont essentiels pour la transformation néoplasique.

Il est très intéressant de noter que l'oncogène $v$-erbA a évolué de sa version normale $c$-erbA pour devenir un puissant antagoniste à la fois des récepteurs de T3 et des récepteurs de l'acide rétinoïque. On peut imaginer que ce processus de sélection a permis de doter l'AEV de son pouvoir oncogénique maximal.

Au vu de ses mécanismes d'action, on comprend pourquoi l'oncogène $v$-erbA n'est pas suffisant pour induire à lui seul une transformation néoplasique. On peut cependant considérer qu'il crée dans la cellule une situation très favorable à cette transformation. Sur la figure 2, on voit que l'effet de v-erbA sur 


\section{RÉFÉRENCES}

1. Evans RM. The steroid and thyroid hormone receptor superfamily. Science 1988 ; 240 : 889-95.

2. Graf $\mathrm{T}$, Beug $H$. Role of the $v$-erb.A and $v$-erb $B$ oncogenes of avian crythroblastosis virus in crythroid cell transformation. Cell 1983 ; $34: 7-9$.

3. Gandrillon $O$, Jurdic $P$, Benchaibi $M$, Xiao JH, Ghysdael J, Sarnarut J. Expression of the $v$-erbA oncogenc in chicken cmbryo fibroblasts stimulates their multiplication in vitro and enhances tumor growth in vivo. Cell 1987 ; 49 : 687-97.

4. Gandrillon O, Jurdic P, Pain C, et al. Expression of $v$-erb $A$, an altered nuclcar hormonc receptor, is sufficient to transform erythrocytic cclls in vitro. Cell $1989 ; 58: 115-21$.

5. Sap J, Munoz A, Schmitt J, Stunnenberg H, Vennström B. Repression of transcription mediated at a thyroid hormone response clement by the $v$-erbA oncogene product. Nature $1989 ; 340: 242-4$.

6. Damm K, Thompson CC, Evans RM. Protcin cncoded by $v$-erbA functions as a thyroid-hormonc receptor antagonist. Nature 1989 ; 339 : 593-7.

7. Pain B, Mclet F, Jurdic F, Samarut J. The carbonic anhydrase II gene, a gene regulated by thyroid hormone and crythropoictin, is repressed by the $v$-erbA oncogene in erythrocytic cells. The New Biologist $1990 ; 2$ : 284-94.

8. Sharif M, Privalsky ML. v-erb.A oncogenc function in neoplasia correlates with its ability to repress retinic acid receptor action. Cell 1991 ; 66 : 885-93.

9. Desbois C, Pain B, Guilhot C, et al. verbA oncogenc abrogates growth inhibition of chicken embryo fibroblasts induced by retinoic acid. Oncogene 1991; 6 : 2129-35.

10. Vogt PK, Bos TJ. jun : oncogenc and transcription factor. Adv Cancer Res 1990 ; 55 : $1-55$.

11. Desbois C, Aubert D, Legrand C, Pain B, Samarut J. A novel mechanism of action for $v$-ErbA : abrogation of the inactivation of transcription factor AP- 1 by retinoic acid and thyroid hormone receptors. Cell 1991; 67 : 731-40.

12. Nicholson RC, Mader S, Nagpal S, Leid M, Rochette-Egly C, Chambon P. Negative regulation of the rat stromclysin genc promoter by retinoic acid is mediated by an AP1 binding sitc. $E M B O J 1990 ; 9$ : 4443-54.

13. Schülc R, Rangarajan $P, Y a n g ~ N$, et al. Retinoic acid is a negative regulator of AP-1-responsive genes. Proc Natl Acad Sci USA 1991 ; 88 : 6092-6.

14. Khazaic K, Panayotou G, Aguzzi A, Samarut J, Gazzolo L, Jurdic P. EGF promotes in vivo tumorigenic growth of primary chicken embryo fibroblasts expressing $v$-myc and enhances in vitro transformation by the $v$ erbA oncogenc. Oncogene 1991; $6: 21-8$.

15. Weinberg RA. Tumor suppressor genes.

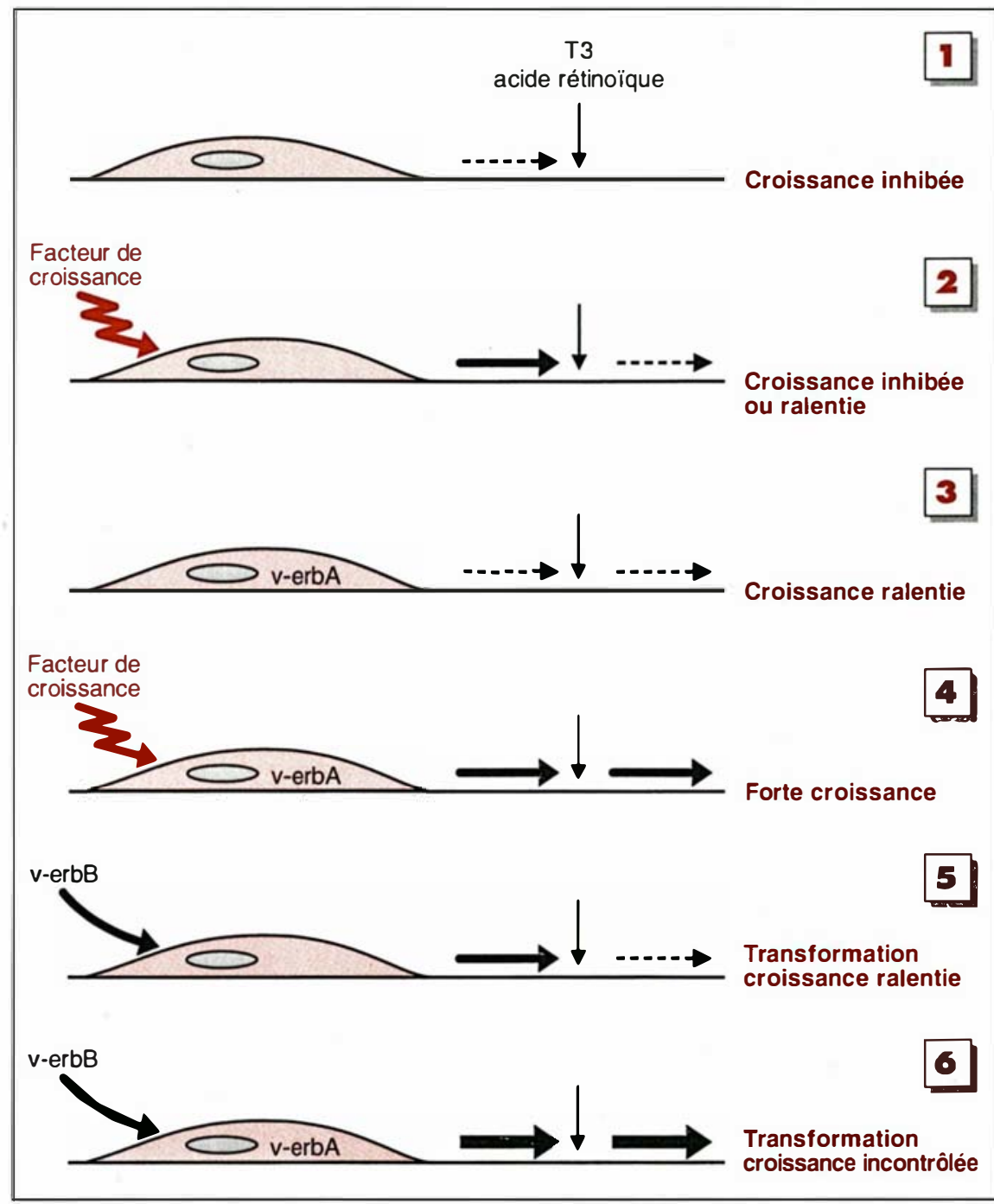

Figure 2. Rôle de l'oncogène v-erbA dans le développement néoplasique. Le schéma représente le comportement d'une cellule normale (situations 1 et 2) et d'une cellule qui exprime l'oncoprotéine v-ErbA (situations 3 à 6). On a supposé, dans ce modèle, que la cellule normale répond à I'hormone T3 ou à l'acide rétinoïque par une inhibition de croissance. Dans les situations 1 et 3, la cellule est cultivée en milieu minimal contenant très peu de facteur de croissance. Dans les situations 5 et 6 , on a imaginé l'intégration de l'oncogène v-erbB dans la cellule. Les flèches horizontales représentent quantitativement l'activité proliférative des cellules.

la prolifération sera d'autant plus marquée que la prolifération de la cellule sera stimulée par ailleurs, dans le meilleur des cas par des facteurs de croissance, au pire, par l'action d'un autre oncogène. C'est ce qui est effectivement observé puisque des fibroblastes qui expriment $v$-erbA montrent une croissance exacerbée en réponse à une stimulation par EGF [14], et un déve- loppement tumoral en réponse à l'oncogène $v$-erbB [3].

Si ces données permettent d'entrevoir le rôle que peuvent jouer certains récepteurs hormonaux dans la transformation cancéreuse, un certain nombre de questions restent posées. En particulier, on ne connaît pas encore les mécanismes moléculaires de l'interférence fonctionnelle entre les récepteurs 
de $\mathrm{T} 3$ et de l'acide rétinoïque et, AP-1 d'une part, et v-ErbA, d'autre part. On imagine que toutes ces protéines interagissent physiquement entre elles, comme cela a été montré pour les protéines c-Fos, c-Jun et le récepteur des glucocorticoïdes $\left(\mathrm{m} / \mathrm{s} n^{\circ} 10\right.$, vol. 6, p. 1003), mais les complexes d'interaction n'ont pas encore été mis en évidence dans les cellules. Par ailleurs, le modèle proposé dans la figure 1 est vraisemblablement encore simpliste. En effet, le dégré d'interaction fonctionnelle entre AP-1 et les récepteurs dépend probablement du contexte créé par la nature et l'état physiologique des cellules. On peut enfin supposer que ce modèle s'applique aussi à d'autres facteurs de transcription apparentés ou non à AP-1. Néanmoins, ce modèle fournit une base de réflexion pour analyser le rôle d'autres oncogènes dans la transformation néoplasique. Compte tenu du rôle joué par les récepteurs de T3 et de l'acide rétinoïque dans la différenciation et la prolifération de certaines cellules, on peut se demander si les gènes qui codent pour ces récepteurs ne peuvent être considérés comme des antioncogènes [15]. L'identification de cancers humains causés par le dysfonctionnement de ces deux types de récepteurs permettra sans doute de répondre à cette question

\section{Christelle Desbois}

Boursier ARC, immuno-virologie moléculaire et cellulaire, université Claude-Bernard Lyon I, Cnrs UMR30, faculté de médecine AlexisCarrel, 69372 Lyon Cedex 08, France.

Denise Aubert

Boursier ARC.

Claude Legrand, Bertrand Pain, Jacques Samarut

Laboratoire de biologie moléculaire et cellulaire, Cnrs UMR 49, Inra, école normale supérieure de Lyon, 46, allée d'Italie, 69364 Lyon Cedex 07, France.

TIRÉS A PART

J. Samarut.

$\mathrm{m} / \mathrm{s} n^{\circ} 2$, vol. 8, fónina 92 